\title{
Disturbed microbial ecology in Alzheimer's disease: evidence from the gut microbiota and fecal metabolome
}

Jianxiong $\mathrm{Xi}^{1}$, Ding Ding ${ }^{2,3^{*}}$, Huiwei Zhu ${ }^{1}$, Ruru Wang ${ }^{1}$, Feng Su${ }^{1}$, Wanqing Wu ${ }^{2,3}$, Zhenxu Xiao ${ }^{2,3}$, Xiaoniu Liang ${ }^{2,3}$, Qianhua Zhao ${ }^{2,3}$, Zhen Hong ${ }^{2,3}$, Hua Fu' ${ }^{1}$ and Qianyi Xiao ${ }^{1 *}$ (D

\begin{abstract}
Background: Gut microbiota (GMB) alteration has been reported to influence the Alzheimer's disease (AD) pathogenesis through immune, endocrine, and metabolic pathways. This study aims to investigate metabolic output of the dysbiosis of GMB in AD pathogenesis. In this study, the fecal microbiota and metabolome from $21 \mathrm{AD}$ participants and 44 cognitively normal control participants were measured. Untargeted GMB taxa was analyzed through 165 ribosomal RNA gene profiling based on next-generation sequencing and fecal metabolites were quantified by using ultrahigh performance liquid chromatography-mass spectrometry (UPLC-MS).

Results: Our analysis revealed that AD was characterized by 15 altered gut bacterial genera, of which 46.7\% (7/15 general) was significantly associated with a series of metabolite markers. The predicted metabolic profile of altered gut microbial composition included steroid hormone biosynthesis, N-Acyl amino acid metabolism and piperidine metabolism. Moreover, a combination of 2 gut bacterial genera (Faecalibacterium and Pseudomonas) and 4 metabolites (N-Docosahexaenoyl GABA, 19-Oxoandrost-4-ene-3,17-dione, Trigofoenoside F and 22-Angeloylbarringtogenol C) was able to discriminate AD from NC with AUC of 0.955 in these 65 subjects.

Conclusions: These findings demonstrate that gut microbial alterations and related metabolic output changes may be associated with pathogenesis of AD, and suggest that fecal markers might be used as a non-invasive examination to assist screening and diagnosis of AD.
\end{abstract}

Keywords: Alzheimer's disease, Gut microbiota, Fecal metabolome, Inflammatory cytokines, Fecal markers

\section{Background}

Alzheimer's disease (AD) is the most prevalent neurodegenerative disorder and is characterized by extracellular plaques composed of amyloid- $\beta$ (A $\beta)$ peptide and intracellular neurofibrillary tangles composed of hyperphosphorylated tau protein [1]. Emerging evidences show that dysbiosis and alterations of the intestinal microbiota

\footnotetext{
* Correspondence: dingding@huashan.org.cn; xiaogianyi@fudan.edu.cn ${ }^{2}$ Institute of Neurology, Huashan Hospital, Fudan University, Shanghai 200040, China

'Department of Preventive Medicine and Health Education, School of Public Health, Fudan University, Shanghai 200032, China

Full list of author information is available at the end of the article
}

contribute to the development of neurodegenerative diseases, including Parkinson' $\mathrm{s}$ disease, schizophrenia, stroke, epilepsy and depression via the "microbiota-gutbrain" axis [2-7]. A few studies have revealed the relationship between gut microbiota (GMB) and AD. Decreased diversity in microbiota is reported in $A D$ and mild cognitive impairment (MCI) patients compared with normal controls, and changes in intestinal microbiota could be used for early detection of $\mathrm{AD}$ [8-10]. Some populations of enterotype bacteria are differential between demented and non-demented dementia [11]. A recent study shows that the fecal and blood microbiota

(c) The Author(s). 2021 Open Access This article is licensed under a Creative Commons Attribution 4.0 International License, which permits use, sharing, adaptation, distribution and reproduction in any medium or format, as long as you give appropriate credit to the original author(s) and the source, provide a link to the Creative Commons licence, and indicate if changes were made. The images or other third party material in this article are included in the article's Creative Commons licence, unless indicated otherwise in a credit line to the material. If material is not included in the article's Creative Commons licence and your intended use is not permitted by statutory regulation or exceeds the permitted use, you will need to obtain permission directly from the copyright holder. To view a copy of this licence, visit http://creativecommons.org/licenses/by/4.0/ The Creative Commons Public Domain Dedication waiver (http://creativecommons.org/publicdomain/zero/1.0/) applies to the data made available in this article, unless otherwise stated in a credit line to the data. 
in MCI patients are similar to that of AD patients compared with normal controls, suggesting the bacterial dysbiosis preceded the onset of dementia [12]. Fecal microbiota transplantation in mice also supports a possible connection between the GMB and AD [13, 14].

The clarification of the gut-brain axis in $\mathrm{AD}$ pathogenesis would provide accessible markers from feces. Human intestinal tract harbors a complex community of microbes with the vast majority of the resident microbial population [15], which plays an important role in the maintenance of human health, including energy extraction, vitamin biosynthesis, prevention of pathogen overgrowth and education of the immune system [16]. Several studies suggest that GMB impact brain through a bidirectional communication system that is connected via neural, immune, endocrine, and metabolic pathways [17-20]. Although 16S ribosomal RNA (rRNA) gene sequencing allows for profiling of complex microbial composition and indicating the microbial entities [21], annotation and the actual microbial activity of GMB is sparse as 16S rRNA gene sequence only provides a global composition analysis but cannot distinguish alive and dead microbes [22]. Fecal metabolites, served as the metabolic output of both GMB and cellular metabolism occurring inside the human intestinal tract, could complement sequencing-based approaches with showing functional readout of microbial activity [23]. Recent evidence indicated that the compounds secreted or influenced by bacteria may cause systemic inflammatory reactions, and then damage the blood-brain barrier (BBB) and promote neurodegeneration [3, 24, 25]. A recent study reported that during $A D$ progression, the alteration of GMB composition leads to the peripheral accumulation of phenylalanine and isoleucine and the increase of pro-inflammatory $\mathrm{T}$ helper 1 cell frequency in the blood in $\mathrm{AD}$ mouse models, which were also observed in MCI due to AD in human [26]. Based on these findings, we sought to investigate potentially $\mathrm{AD}$ relevant gut microbes and their metabolic outputs [27], which has rarely been reported in AD.

Therefore, in this study, we investigated the altered GMB composition and metabolic changes in the fecal samples that are relevant with $\mathrm{AD}$, and examined their correlation by integrating the two omics findings. Based on the correlation analysis, we determined the potentially functional microbiota and its metabolic output, and further identified the potentially fecal markers of AD. Considering that compounds influenced by bacteria may cause systemic inflammatory reactions, and the altered cytokine levels had been reported as potential hallmark of $\mathrm{AD}$, we also analyzed the association between differential fecal markers and peripheral inflammatory cytokines that linked with $\mathrm{AD}$ to further understand the function of altered GMB composition.

\section{Results}

Characteristics of study subjects

The demographic and clinical information for $21 \mathrm{AD}$ participants and 44 cognitively normal control (NC) participants was presented in Table 1 . No statistically significant difference in proportion of sex was found $(P<$ 0.05 ) between $\mathrm{AD}$ group and $\mathrm{NC}$ group. $\mathrm{AD}$ and $\mathrm{NC}$ groups also did not differ with respect to age at examination, education years and body mass index (BMI) $(P<$ 0.05). The mean MMSE score was significantly lower in AD participants (18.0) than in NC participants (29.0), $P<0.001$. APOE $\& 4$ carrier rate was significantly higher in $\mathrm{AD}$ participants $(50.0 \%)$ than in $\mathrm{NC}$ participants (11.9\%), $P<0.001$.

\section{Genera of GMB between AD and NC participants}

After quality filtering and trimming, sequencing of the V3-V4 region of 16S rRNA gene generated 3.55 million sequence reads from 65 fecal samples with an average length of 419 . At the $97 \%$ sequence similarity level, a total of 1014 OTUs were obtained and matched to 13 phyla, 20 classes, 35 orders, 71 families and 251 genera. The Venn diagram showed 53 unique OTUs in AD, 185 unique OTUs in NC and 776 shared OTUs between two groups (Additional file 1: Fig. S1A).

The $\alpha$-diversity did not differ between AD group and NC group (Additional file 2: Table S1). With respect to $\beta$-diversity, principal co-ordinates (PCoA) based on Bray-Curtis dissimilarity showed the significant differences of GMB composition between the fecal samples from $\mathrm{AD}$ participants and that from $\mathrm{NC}$ participants (PERMANOVA, $R^{2}=0.025, P=0.027$ ) (Fig. 1a). Meanwhile, control analyses were performed to determine whether the potentially confounding variables influenced this global microbial phenotype [28]. The results showed that the $\mathrm{AD}$ participants or $\mathrm{NC}$ participants were not clustered based on sex (Additional file 1: Fig. S1B), age

Table 1 Clinical characteristics of AD subjects and cognitively normal control (NC) subjects

\begin{tabular}{llll}
\hline Baseline characteristic & AD & NC & $\boldsymbol{P}_{\text {value }}$ \\
\hline Participants, n & 21 & 44 & \\
Age, years (IQR) & $76.2(9.9)$ & $78.4(6.6)$ & 0.262 \\
Female, n (\%) & $8(38.1)$ & $24(54.6)$ & 0.215 \\
Education, years (IQR) & $12(3)$ & $12(7)$ & 0.889 \\
MMSE scores, mean (IQR) & $18(8)$ & $29(2)$ & $<0.001$ \\
APOE \&4, n (\%) & $10(50.0)^{\mathrm{b}}$ & $5(11.9)^{\mathrm{c}}$ & $<0.001$ \\
BMI, mean (IQR) & $22.8(3)$ & $23.1(5)$ & 0.388 \\
\hline
\end{tabular}

Values are shown as median (interquartile range, IQR) or number (percent) $B M I$ Body mass index, $A D$ Alzheimer's disease, $N C$ cognitively normal control ${ }^{a}$ Mann-Whitney $U$ test or $x^{2}$ test

${ }^{b}$ Missing data of $A P O E$ genotype $(n=1)$

c Missing data of $A P O E$ genotype $(n=2)$ 


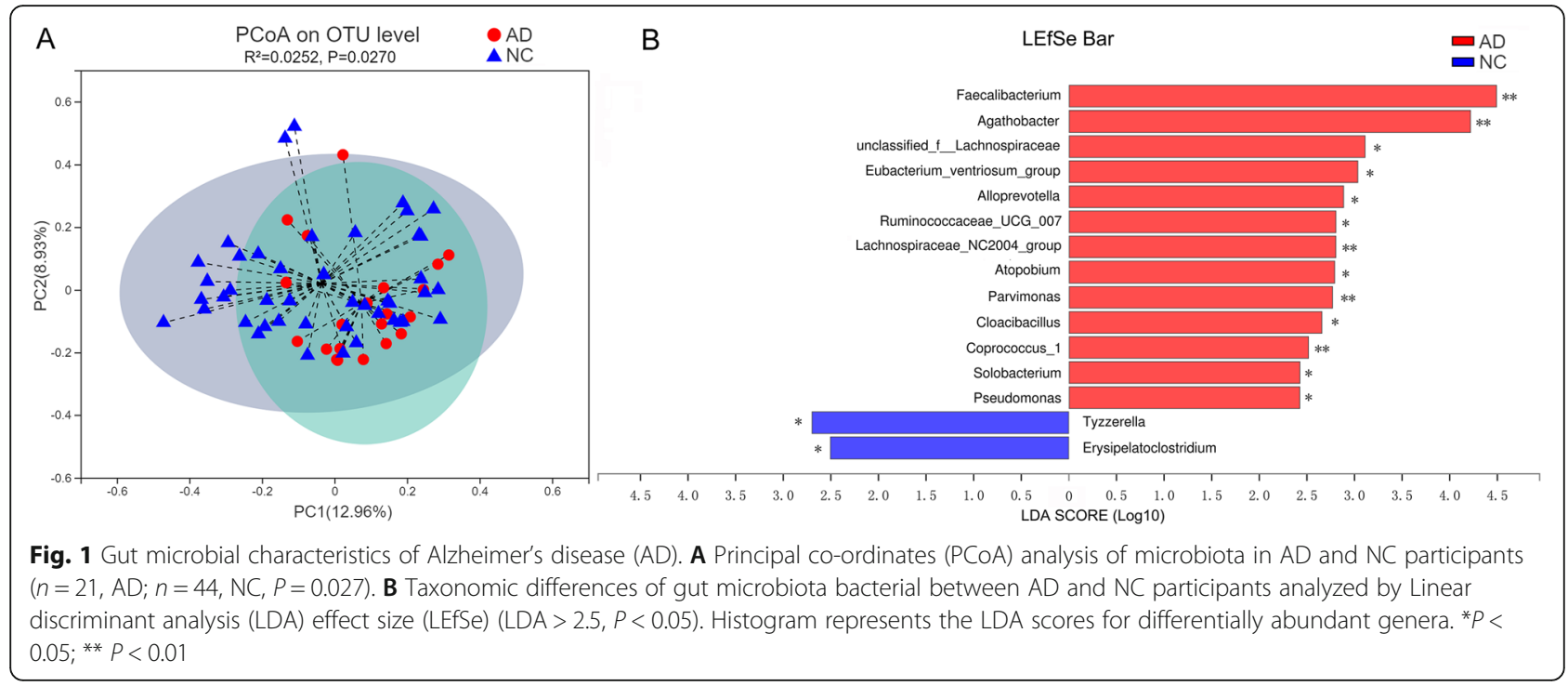

(Additional file 1: Fig. S1C-D) or BMI (Additional file 1: Fig. S1E-F).

Furthermore, LEfSe analysis (Fig. 1b) showed that 15 genera significantly differed between $\mathrm{AD}$ group and $\mathrm{NC}$ group (LDA values $>2.5$, Wilcoxon rank-sum test, $P<$ 0.05; Additional file 3: Table S2). Specifically, AD participants exhibited 13 increased genera compared to NC participants, which belonged to bacterial families Lachnospiraceae (genera Agathobacter, unclassified_f_Lachnospiraceae, Eubacterium_ventriosum_group, Lachnospiraceae_NC2004 and Coprococcus_1), Ruminococcaceae (genera Faecalibacterium and Ruminococcaceae_UCG-007), Prevotellaceae (genus Alloprevotella), Atopobiaceae (genus Atopobium), Clostridiales (genus Parvimonas), Synergistaceae (genus Cloacibacillus), Erysipelotrichaceae (genus Solobacterium) and Pseudomonadaceae (genus Pseudomonas). In contrast, $\mathrm{AD}$ participants exhibited 2 decreased genera compared to NC participants, which belonged to bacterial families Lachnospiraceae (genus Tyzzerella) and Erysipelotrichaceae (genus Erysipelatoclostridium). At phyla level, these 15 differential bacterial genera were classified to Firmicutes $(11 / 15,73.3 \%)$, Bacteroidetes (1/15, 6.7\%), Actinobacteria $(1 / 15,6.7 \%)$, Proteobacteria $(1 / 15,6.7 \%)$ and Synergistetes $(1 / 15,6.7 \%)$.

\section{Fecal Metabolome between AD and NC participants}

Fecal metabolites, served as the metabolic output of both GMB and cellular metabolism occurring inside the human intestinal tract, is considered a functional readout of the GMB. As show in Fig. 2a, the OPLS-DA scores plot showed a clear variation between $\mathrm{AD}$ group and $\mathrm{NC}$ group (Permutation test: $R^{2} Y=0.70, Q^{2} Y=-0.28$ for positive ion, and $R^{2} Y=0.74, Q^{2} Y=-0.15$ for negative ion). Metabolome of $\mathrm{AD}$ participants showed 15 differential fecal metabolites relative to the Control group $(P<0.05$, VIP $>1$, Table 2$)$, with decreased levels of 11 metabolites and increased level of 4 metabolites in AD. The implicated metabolites belonged to organic acids (3 metabolites: N-Docosahexaenoyl-GABA; Hypoglycin $B$ and 12-Hydroxydodecanoic acid), lipids and lipid-like molecules (7 metabolites: 19-Oxoandrost-4ene-3,17-dione; 5-Butyl-3,4-dimethyl-2-furanundecanoic acid; Trigofoenoside F; Sagittariol; 22Angeloylbarringtogenol C; $1 \alpha, 25$-dihydroxy-3 $\alpha$-methyl3-deoxyvitamin D3 and PG(16:0/0:0)[U]), Benzenoids (2 metabolites: (4E)-12-hydroxy-1-(4-hydroxy-3-methoxyphenyl)dodec-4-en-3-one and 5-3', 5'-Dihydroxyphenyl$\gamma$-valerolactone), Organic nitrogen (N,N-Dimethylsphingosine), Organoheterocyclic $((5 \alpha, 8 \beta, 9 \beta)-5,9$-Epoxy-3,6megastigmadien-8-ol) and Piperidine (1-ACETYLPIPERIDINE) (Table 2). KEGG pathway enrichment analysis revealed that the differentially expressed metabolites were involved in steroid hormone biosynthesis (19Oxoandrost-4-ene-3,17-dione), N-Acyl amino acid metabolism (N-Docosahexaenoyl-GABA) and Piperidine metabolism (1-ACETYLPIPERIDINE) (Table 2).

Relationship between altered gut microbial genera, fecal metabolites, clinical characteristics and inflammatory cytokines linked with AD

As shown in Fig. 2b, the Pearson correlation analyses indicated that $46.7 \%$ (7/15 genera) of altered bacterial genera were significantly associated with a series of altered fecal metabolites $(P<0.05, r>0.30$ or $<-0.30$ [29], associated with more than one metabolite), suggesting a general correlation between gut bacterial file and metabolites. The coefficient of correlation and the $P$ value between bacterial genera and fecal metabolites were presented in Additional file 4: Table S3 and 


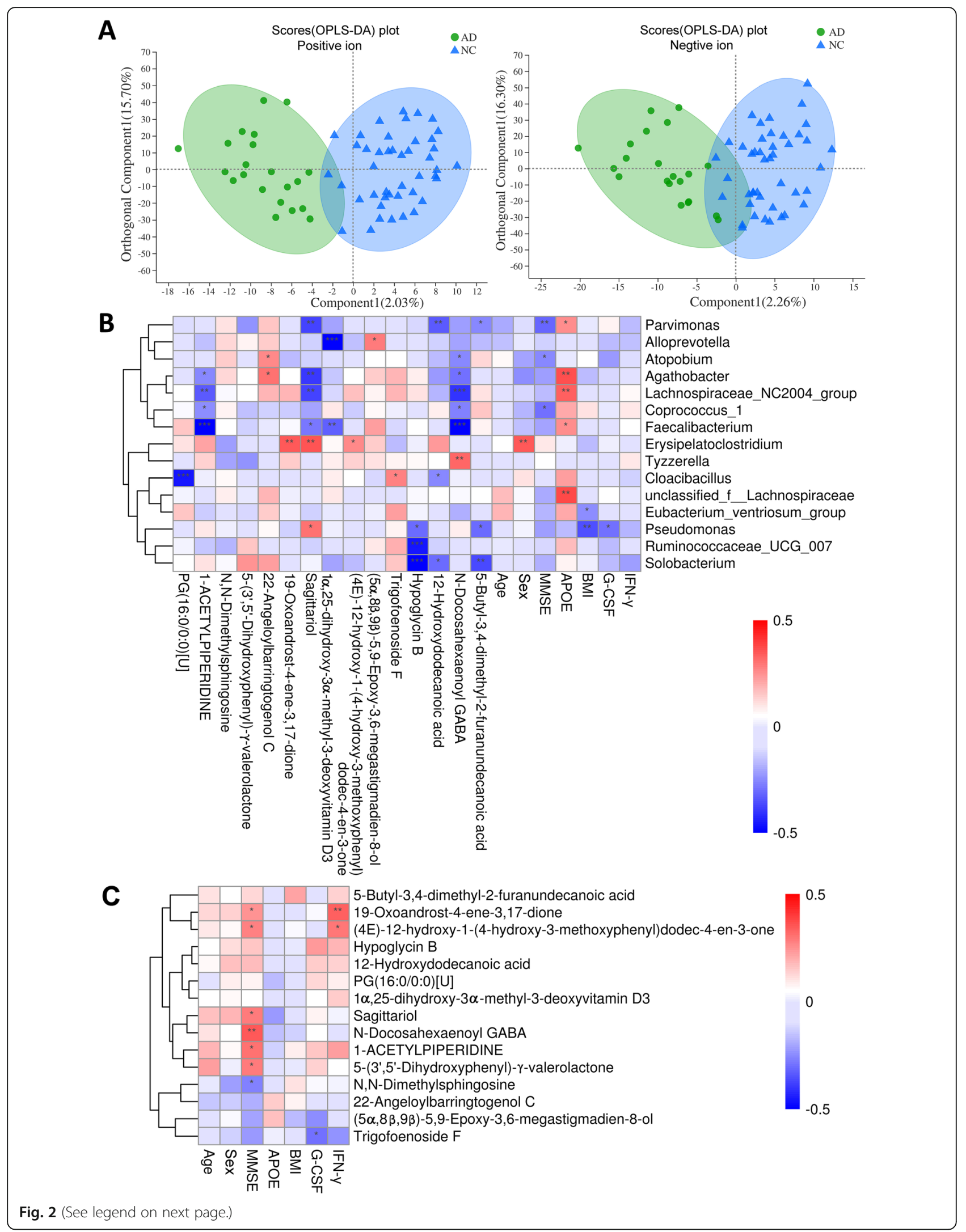


(See figure on previous page.)

Fig. 2 Characteristics of metabolites between AD participants and NC participants and the correlation analyses. A The orthogonal partial leastsquares discriminant analysis (OPLS-DA) plot of fecal metabolites in comparisons of AD and NC groups (left: positive ion; right: negative ion). $\mathbf{B}$ Pearson correlation between 15 most differential genera and 15 differential metabolites, inflammatory cytokines, clinical characteristics linked with AD. The results were presented as a heatmap. Red squares indicate positive associations; blue squares indicate negative associations. ${ }^{*} P<0.05,{ }^{* *}$ $P<0.01$, *** $P<0.001$ denoted statistical significance. C Associations of fecal metabolites with inflammatory cytokines and clinical parameters. Red squares indicate positive associations; blue squares indicate negative associations. ${ }^{*} P<0.05$; ${ }^{* *} P<0.01$

Additional file 5: Table S4, respectively. These genera mainly belonged to Lachnospiraceae (Lachnospiraceae NC2004 and Agathobacter), Erysipelotrichaceae (Erysipelatoclostridium and Solobacterium), Ruminococcaceae (Faecalibacterium), Pseudomonadaceae (Pseudomonas) and Clostridiales (Parvimonas) at family level. Additionally, significant correlations were observed between some gut microbial genera and clinical parameters including sex, BMI, MMSE score, APOE genotype and GCSF (Fig. 2b). These findings suggested that AD was simultaneously distinguished by changes in GMB and fecal metabolome.

As shown in Table 3, AD participants presented significantly decreased expression of serum G-CSF and IFN- $\gamma$ compared to NC participants $(P=0.008$ and $P=$ 0.007 , respectively) (Table 3 ). A significant negative correlation of Trigofoenoside F metabolite with G-CSF ( $r=$ $-0.301, P=0.019)$ and a significant positive correlation of 19-Oxoandrost-4-ene-3,17-dione metabolite with IFN- $\gamma(r=0.339, P=0.006)$ were observed (Fig. 2c, Additional file 6: Table S5 and Additional file 7: Table S6).

Table 2 Alteration of Metabolites in AD subjects compared with cognitively normal controls

\begin{tabular}{|c|c|c|c|c|c|}
\hline Compound & Metabolite & $\begin{array}{l}\text { Predicted molecular pathways or biological } \\
\text { functions }^{\mathrm{a}}\end{array}$ & VIP & $\begin{array}{l}\text { FC } \\
\text { (AD/ } \\
\text { NC) } \\
\end{array}$ & $P$ \\
\hline \multicolumn{6}{|c|}{ Lipids and lipid-like molecules } \\
\hline $\begin{array}{l}\text { Androstane } \\
\text { steroids }\end{array}$ & 19-Oxoandrost-4-ene-3,17-dione & Steroid hormone biosynthesis & 2.4 & 0.73 & 0.023 \\
\hline Fatty acids & 5-Butyl-3,4-dimethyl-2-furanundecanoic acid & Antixoxidants and radical scavengers & 2.1 & 0.80 & 0.034 \\
\hline $\begin{array}{l}\text { Steroidal } \\
\text { glycosides }\end{array}$ & Trigofoenoside F & A compound isolated from fenugreek seeds & 1.5 & 1.07 & 0.011 \\
\hline Triterpenoids & 22-Angeloylbarringtogenol C & A constituent of $C$. sinensis & 3.3 & 1.22 & 0.042 \\
\hline Diterpenoids & Sagittariol & - & 2.6 & 0.87 & 0.042 \\
\hline $\begin{array}{l}\text { Vitamin D } \\
\text { derivatives }\end{array}$ & 1a,25-dihydroxy-3a-methyl-3-deoxyvitamin D3 & - & 2.7 & 0.87 & 0.031 \\
\hline- & $P G(16: 0 / 0: 0)[U]$ & - & 1.3 & 0.95 & 0.019 \\
\hline \multicolumn{6}{|c|}{ Organic acids and derivatives } \\
\hline Amino acids & N-Docosahexaenoyl GABA & N-Acyl amino acid metabolism & 2.3 & 0.81 & 0.001 \\
\hline Hydroxy acids & 12-Hydroxydodecanoic acid & $\begin{array}{l}\text { Substrate of glutathione-dependent formaldehyde } \\
\text { dehydrogenase }\end{array}$ & 1.6 & 0.85 & 0.047 \\
\hline Amino acids & Hypoglycin B & Natural toxins & 1.4 & 0.86 & 0.037 \\
\hline \multicolumn{6}{|l|}{ Benzenoids } \\
\hline Methoxyphenols & $\begin{array}{l}\text { (4E)-12-hydroxy-1-(4-hydroxy-3- } \\
\text { methoxyphenyl)dodec-4-en-3-one }\end{array}$ & A predicted metabolite generated by BioTransformer & 2.1 & 0.67 & 0.020 \\
\hline Benzenediols & 5-(3',5'-Dihydroxyphenyl)- $\gamma$-valerolactone & $\begin{array}{l}\text { A tea polyphenol metabolite detected in biological } \\
\text { fluids }\end{array}$ & 2.3 & 0.73 & 0.041 \\
\hline \multicolumn{6}{|c|}{ Organic nitrogen compounds } \\
\hline Amines & $\mathrm{N}, \mathrm{N}$-Dimethylsphingosine & Regulation of sphingolipid-mediated functions & 3.9 & 1.35 & 0.032 \\
\hline \multicolumn{6}{|c|}{ Organoheterocyclic compounds } \\
\hline Benzopyrans & 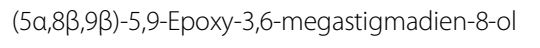 & - & 1.8 & 1.23 & 0.045 \\
\hline \multicolumn{6}{|l|}{ Piperidine } \\
\hline Piperidine & 1-ACETYLPIPERIDINE & Piperidine metabolism & 1.9 & 0.80 & 0.041 \\
\hline
\end{tabular}

$A D$ Alzheimer's disease, $N C$ cognitively normal control, VIP variable importance in the projection, $F C$ fold change

${ }^{a}$ Information referencing KEGG pathway database and HDBM database 
Table 3 Association of peripheral inflammatory cytokines and Alzheimer's disease risk

\begin{tabular}{|c|c|c|c|c|c|c|}
\hline \multirow[t]{2}{*}{ Cytokines } & \multirow{2}{*}{$\begin{array}{l}\text { Number of subjects } \\
A D / N C\end{array}$} & \multicolumn{2}{|c|}{ Mean } & \multicolumn{2}{|c|}{ Median } & \multirow{2}{*}{$\begin{array}{l}P \\
\text { value }\end{array}$} \\
\hline & & $A D$ & NC & $A D$ & $\mathrm{NC}$ & \\
\hline IL-6 & $18 / 41$ & 3.57 & 2.75 & 1.44 & 1.63 & 0.792 \\
\hline IL-8 & $18 / 43$ & 7.38 & 6.77 & 6.09 & 5.99 & 0.794 \\
\hline IL-10 & $18 / 43$ & 1.08 & 1.85 & 1.01 & 1.18 & 0.315 \\
\hline G-CSF & $19 / 43$ & 1.28 & 1.84 & 1.27 & 1.59 & 0.008 \\
\hline IL-7 & $19 / 43$ & 2.14 & 2.22 & 2.05 & 2.05 & 0.667 \\
\hline |L-8 & $19 / 43$ & 3.17 & 3.29 & 2.40 & 3.15 & 0.113 \\
\hline IL-12 & $19 / 42$ & 1.32 & 1.50 & 0.95 & 0.95 & 0.849 \\
\hline $\mathrm{IFN}-\gamma$ & $19 / 42$ & 0.97 & 1.75 & 0.66 & 1.40 & 0.007 \\
\hline MCP-1 & $19 / 43$ & 6.87 & 8.27 & 6.85 & 7.72 & 0.172 \\
\hline MIP-1 $\beta$ & $19 / 43$ & 8.33 & 10.95 & 6.45 & 8.28 & 0.194 \\
\hline TNF-a & $19 / 39$ & 2.27 & 2.67 & 1.61 & 2.12 & 0.314 \\
\hline
\end{tabular}

$A D$ Alzheimer's disease, $N C$ cognitively normal control

${ }^{a}$ Comparison between AD group and NC group of median, using MannWhitney $\mathrm{U}$ test

These findings suggested that some fecal metabolites might affect systemic inflammatory reactions during AD development.

\section{Potential contribution of fecal markers of AD}

Among the newly implicated 17 fecal markers in our study, including of differential 7 fecal genera and their 8 significantly correlated fecal metabolites, and 2 fecal metabolites that were correlated with differential inflammatory cytokines in $\mathrm{AD}$, combination of 6 fecal markers, including of 2 genera (Faecalibacterium and
Pseudomonas) and 4 metabolites (N-Docosahexaenoyl GABA, 19-Oxoandrost-4-ene-3,17-dione, Trigofoenoside $\mathrm{F}$ and 22-Angeloylbarringtogenol $\mathrm{C}$ ), was able to discriminate AD from $\mathrm{NC}$ with an AUC of 0.955 in these 65 subjects. The AUC of each separate microbial $(\mathrm{AUC}=0.798)$ or metabolic markers $(\mathrm{AUC}=0.873)$ was significantly lower than that of combination of microbial and metabolic markers, $P<0.001$ and $P=0.033$, respectively (Fig. 3). Addition of inflammatory cytokines did not significantly improve the discriminative power of the combined model $(P=0.857)$.

\section{Discussion}

This study demonstrated that AD exhibited the disorder in microbial ecology, which may affect both metabolism and subsequent peripheral inflammatory cytokine in human subjects. Importantly, our study showed that the AUC for discriminating AD from NC was 0.955 for combination of fecal microbial markers and correlated metabolites (a total of six fecal markers), suggesting the potential of fecal markers as a non-invasive tool for screening or assistant diagnosis of AD. Further studies in large scale and longitudinal analysis can be justified to validate and improve the clinical utility of this fecal marker panel.

In our study, $\mathrm{AD}$ was characterized by increased 13 genera, of which 5 genera belonged to family Lachnospiraceae. Our results confirmed the previous report by chi et al. that the higher abundance of family Lachnospiraceae (general Fusicatenibacter, Blautia, and Dorea) was negatively associated with MMSE score [30]. In

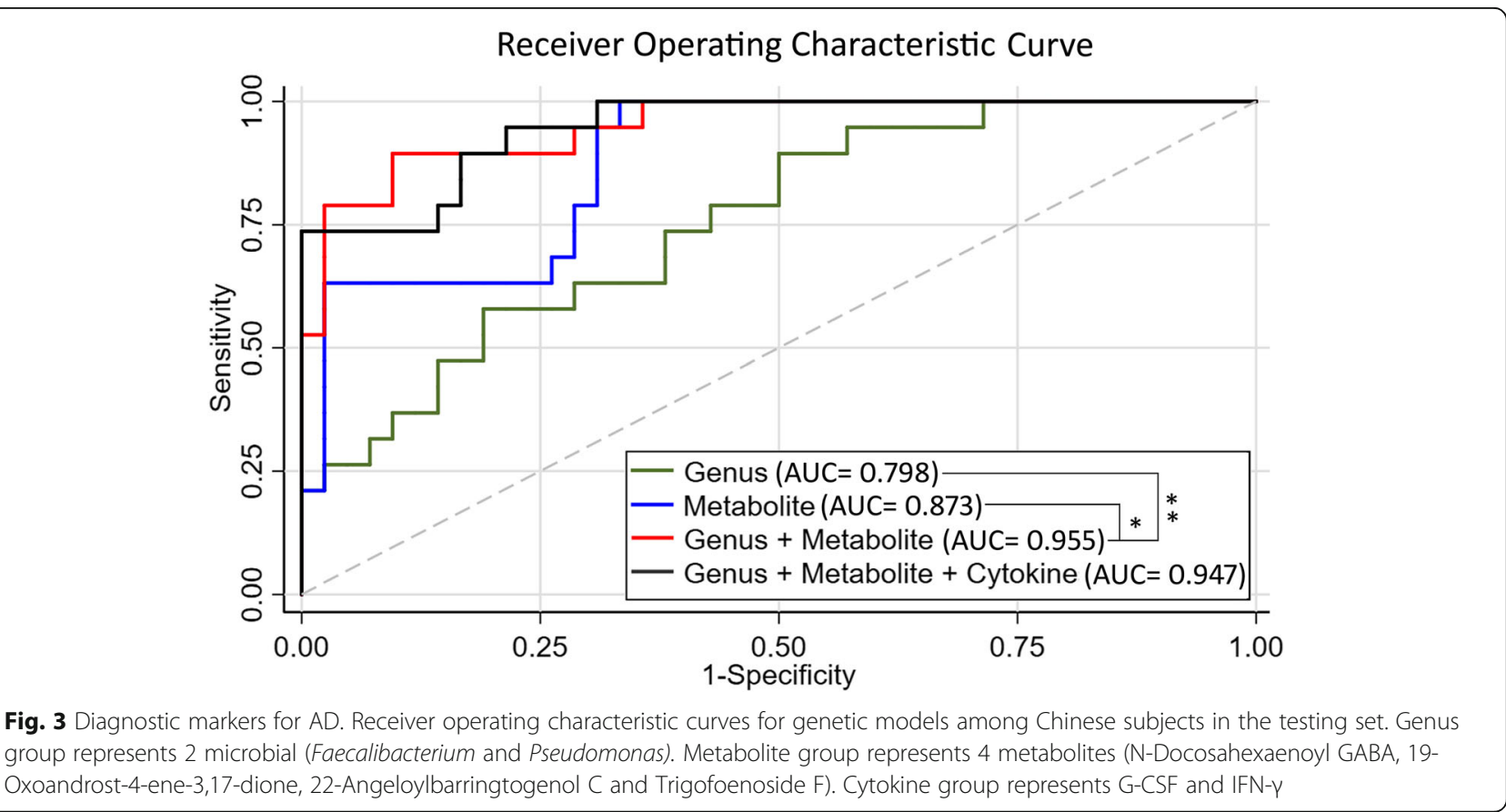


addition, a metagenomic study indicated the association of the family Lachnospiraceae with Type 2 diabetes and the intestinal colonization by the Lachnospiraceae contributes to the development of diabetes [31]. It is well known that diabetes and insulin resistance were risk factors of AD [32-34], therefore, a potential mechanism by which gut Lachnospiraceae influences $\mathrm{AD}$ development might be resulted from promoting the development of diabetes.

Our results showed that the abundance of genera Pseudomonas and Faecalibacterium increased in AD participants and these two genera contributed to fecal marker panel for effectively discrimination of $\mathrm{AD}$ from NC (Fig. 3). The genus Pseudomonas was found to be a possible risk factor of $\mathrm{AD}$, as it increased both in fecal sample (our study results) and blood sample [12] from AD subjects, compared with that in cognitive normal controls. Functional studies reported that $P$. aeruginosa infection could increase endothelial tau phosphorylation and permeability [35, 36], which is a common pathophysiological mechanism in $\mathrm{AD}$ pathogenesis. In addition to the pathogenic $P$. aeruginosa strain, the genus Pseudomonas also comprises non-pahtogenic strains, such as $P$. syringae strain [37]. So far, little is known about how interactions between different pathogenic and non-pathogenic Pseudomonas strains within the host impact. Future studies should furtherly identify the specific pathogenic strain responsible to AD and explore the interaction between pathogenic and nonpathogenic strains on the occurrence of AD. With regard to Faecalibacterium, F. prausnitzii (F. prausnitzii) is the founding strain and has been studied extensively. $F$. prausnitzii plays a beneficial role in anti-inflammatory and promoting gut health $[38,39]$. Several studies reported lower abundance of $F$. prausnitzii in patients with inflammatory bowel diseases [40,41], type 2 diabetes [42] and colon cancer [43]. However, higher abundance in $F$. prausnitzii was reported in pediatric patients with Crohn's Disease compared with controls [44, 45], indicating the detrimental role of $F$. prausnitzii, at least in some contexts. Lopez-Siles et al. [46] has found that patients with Crohn's Disease may harbor a unique set of F. prausnitzii OTUs, as compared with controls. A study with deep metaproteomics approach has defined five available strain proteomes of $F$. prausnitzii. Therefore, inconsistent role of $F$. prausnitzii may be due to the different function of different strains of Faecalibacterium genera, which might be the potential confounders for the ROC curves evaluated in our study. Future research is needed to determine the specific strains that relevant to AD. Our results reported a higher abundance of gut Faecalibacterium in AD than NC group, providing additional evidence of detrimental role of Faecalibacterium. One explanation of may be that Faecalibacterium could reduce secretion of proinflammatory cytokines IFN- $\gamma$ [38]. Then, reduced IFN- $\gamma$ causes increase of $A \beta$ deposits [47]. In fact, we also found the lower peripheral IFN- $\gamma$ expression in AD compared with NC group, which supports this hypothetical explanation.

AD participants in our study showed alteration of fecal metabolome compared to NC participants. Correlation analysis provided a significant association of altered bacterial genera with altered metabolites. Furthermore, pathway analysis linked the alteration of metabolites to three metabolic pathways including Steroid hormone biosynthesis, N-Acyl amino acid metabolism and Piperidine metabolism. Together, these findings suggest the potential mechanism of GMB affecting occurrence or development of AD. Future functional studies are justified to formally verify that if and how these metabolic pathways participate in the occurrence or development of AD. Notably, among 15 altered metabolites in AD, 7 metabolites belong to lipid or lipid-like molecules, suggesting that lipid-mediated metabolic or synthetic processes in gut are linked with AD. The 19-Oxoandrost-4ene-3, 17-dione, a decreased lipid-like molecular in $\mathrm{AD}$ compared with NC group in our study, was involved in estrogen biosynthesis. Estrogen is a potent factor that not only prevents vascular disease but also plays an important role in the preservation of neurons and reparation of neurons damaged by the disease [48]. A population-based study indicated higher frequency of estrogen use in non-patients compared with Alzheimer's disease patients [49]. Thus, decreased 19-Oxoandrost-4ene-3, 17-dione in gut may influence the $\mathrm{AD}$ pathology by downregulating the estrogen. 5-Butyl-3, 4-dimethyl2-furanundecanoic acid, another decreased lipid-like metabolite in $\mathrm{AD}$, is a furan fatty acid (F-acid). F-acids play an protective role for Alzheimer's disease progression mainly by preventing lipid peroxidation $[50,51]$ and protecting polyunsaturated fatty acids (PUFAs) [52], suggesting possible mechanism for lower level of 5-Butyl-3, 4-dimethyl-2-furanundecanoic acid in $\mathrm{AD}$ than $\mathrm{NC}$ group. Regarding to amino acid metabolism, all three metabolites belonging to organic acids showed decreased level in $\mathrm{AD}$, among which N-Docosahexaenoyl GABA was annotated to be involved in N-Acyl amino acid metabolism. Consistent with our results, N-acyl amino acids have anti-inflammatory and neuroprotective effects [53], but their biosynthesis and catabolism have not been fully elucidated [54].

Additionally, we observed decreased serum G-CSF and IFN- $\gamma$ level in AD participants compared with NC participants. The consistent results were also observed in previous clinical and animal studies. Decreased plasma levels of G-CSF were previously reported in patients with early $\mathrm{AD}$ in comparison with healthy controls [55]. Moreover, in AD participants, G-CSF levels showed a 
significant inverse correlation with amyloid- $\beta(A \beta 1-42)$ levels in cerebrospinal fluid [55]. Mouse model experiment proved that subcutaneous administration of G-CSF into two different $A \beta$-induced $A D$ mouse models could substantially rescue their cognitive/memory functions [56]. Additionally, in the stroke mouse model, administration of G-CSF could lead to neurogenesis near the injured area and the neurological and functional recovery [57-59]. With respect to IFN- $\gamma$, although the relationship between IFN- $\gamma$ level and AD has not been reported, IFN- $\gamma$ overexpression was found to reduce $A \beta$ deposits and peripheral monocytes infiltration in amyloid precursor protein (APP) transgenic mice [47]. Additionally, in both 5XFAD and APP/PS1 mice model at intermediate stage of $A D$, reversion of decreased IFN- $\gamma$ level by transient depletion of Treg cells could increase infiltration and recruitment of leukocyte to $A \beta$ plaques and then alleviate the AD pathology [60]. The findings of these functional studies support our observation that IFN- $\gamma$ is decreased in $\mathrm{AD}$ compared with $\mathrm{NC}$ group.

By integrating the correlation analyses of three groups of markers (GMB, fecal metabolites and peripheral inflammatory cytokines), we found two potential mechanistic pathways for the activity and function of GMB in $\mathrm{AD}$ pathogenesis. One pathway was that decrease of 19Oxoandrost-4-ene-(3,17)-dione metabolite caused by low abundance of gut Erysipelatoclostridium might lead to reduction of peripheral IFN- $\gamma$ level, which subsequently influenced AD pathogenesis. There are some evidences supporting the potential of this mechanism.19Oxoandrost-4-ene-(3,17)-dione was involved in estrogen biosynthesis as we discussed above, besides, administration of estrogen to mouse could enhance the secretion of IFN- $\gamma$ by mouse spleen cells [61] and by CD4+ human $\mathrm{T}$ cell clones [62]. The second pathway was that increase of Trigofoenoside $\mathrm{F}$ metabolite caused by high abundance of gut Cloacibacillus might lead to reduction of peripheral G-CSF level and then influenced AD pathogenesis. Though there are no other references supporting this mechanism for now, our findings have provided evidences for better understanding the role of microbiota in AD pathogenesis. It should be admitted that whether and how these mechanisms are involved in the occurrence or development of $\mathrm{AD}$ is still unclear, and further researches are needed.

There are several limitations to this study. First, it was a preliminary study with small sample size, and studies of larger population with different groups across Asia or with different ethnicity would give a better picture of the connection between GMB, metabolites and inflammatory cytokines, and also validate the predictive performance using fecal marker penal. Second, although our study suggests that the GMB and relative metabolic may be associated with the pathogenesis of $\mathrm{AD}$, future intervention study and animal experiments are needed to identify whether this relationship connected by a causal relationship or is a simple incidental association. Third, functional study should be carried out to clarify the "bacterial-metabolite-cytokine" mechanism in $\mathrm{AD}$ pathogenesis and define the specific and effective targets for AD intervention and therapy. Fourth, the diagnosis of AD in this study was based on symptoms without disease severity information, and the neuroimaging markers should be included in further confirmatory studies for accurate diagnosis. Fifth, although we used LEfSe to identify the key bacterial taxa that altered between $\mathrm{AD}$ group and $\mathrm{NC}$ group, there are other methods to screen different bacterial taxa, such as PLS-DA VIP plot [63] or Random Forest analysis [64]. Future researches with follow-up data should be conducted to determine which method is better in determining key bacterial taxa responsible for AD risk. Finally, we performed the binary logistic regression analysis with the ROC curves to select and quantify which combination of fecal markers were best relevant to $\mathrm{AD}$. Larger studies with cross-validation are justified to formally test the hypothesis and assess its predictive performance.

\section{Conclusions}

In conclusion, our study provides preliminary evidence that AD exhibited changes in fecal microbial composition and its metabolic output, and these identified fecal markers enabled discriminating $\mathrm{AD}$ from $\mathrm{NC}$ with high accuracy. These findings improve our understanding of the role of GMB in AD pathogenesis, and provide a non-invasive way for early-stage clinical screening or assistant diagnosis of $\mathrm{AD}$.

\section{Methods \\ Study participants}

From April 2018 to July 2019, 21 patients with AD from memory clinic at the Department of Neurology of Huashan Hospital and 44 cognitively normal controls (NC) from community participants with normal cognition from the Shanghai Aging Study [65] were recruited. These two groups did not differ in age, sex, and education years $(P>0.05$, Table 1$)$.

All participants recruited in study had similar diet habit. Exclusion criteria in this study included: use of antibiotics, probiotics, or prebiotics within a month before sampling; corticosteroid use, immune stimulating medications and immunosuppressive agents; use of antidepressant; gastrointestinal surgery in past 5 years; human immunodeficiency virus or serious bacterial infection in the medical history; inflammatory bowel disease, persistent, infectious gastroenteritis, colitis or gastritis; diarrhea or constipation at sampling. 
The study protocol was approved by the Medical Ethics Committee of Huashan Hospital at Fudan University (IRB\#2009-195) and the Ethics Committee of the Department of Public Health at Fudan University (IRB\#2019-04-0739), Shanghai, China. All participants or participants' legally acceptable representatives provided written informed consent to participate in this study. Subjects in this study were recruited based on the Shanghai Aging Study.

\section{Clinical interview and diagnosis of cognition}

Neurologists conducted face-to-face interview for AD participants and controls to collect the clinical and demographic data. Neurologists and neuropsychologists reviewed the clinical and neuropsychological data and reached a consensus diagnosis of $\mathrm{AD}$ based on the DSM-IV and NINCDS-ADRDA criteria [66, 67]. The detailed information of clinical and neuropsychological assessment and diagnosis procedures were described elsewhere [68]. The genotypes of $A P O E$ were determined using the TaqMan SNP Genotyping assay [69]. APOE $\varepsilon 4$ positive was defined as participants who have at least one $\varepsilon 4$ allele.

\section{Sample collection}

First morning fecal samples were collected in sterile fecal collection containers from each participant at home and packaged with frozen gel packs. Participants returned the fecal collection containers to Huashan hospital within $2 \mathrm{~h}$. Upon receipt, samples were immediately subsampled into prepared sterile tube sand frozen at $-80{ }^{\circ} \mathrm{C}$ until further analysis. In the morning of the same day with fecal sample collection, the overnight fasting blood sample was collected from each participant by research nurses at Huashan Hospital. After centrifugation, serum were subsampled (300ul) into prepared sterile tubes and frozen at $-80^{\circ} \mathrm{C}$ until analysis.

\section{DNA extraction and 16S rRNA gene sequencing}

The E.Z.N.A. ${ }^{@}$ Stool DNA Kit (Omega Bio-Tek, Norcross, GA, USA) was used for microbial DNA extraction of fecal samples and extracted DNA samples were stored in elution buffer (Tris-HCL) provided in kit at $-80^{\circ} \mathrm{C}$ until sequencing. The NanoDrop 2000 UV-vis spectrophotometer (Thermo Scientific, Wilmington, USA) was used for measuring DNA concentration. DNA integrity were shown and assessed by $1 \%$ agarose gel electrophoresis. The V3-V4 region of the $16 \mathrm{~S}$ rRNA gene was amplified using the 6-bp barcoded universal primers: 338 forward (5'-ACTCCTACGGGAGGCAGCAG-3') and 806 reverse (5'-GGACTACHVGGGTWTCTAAT-3'). PCR reaction was carried out in a $20 \mu \mathrm{L}$ reaction volumes containing $5 \times$ FastPfu Buffer $(4 \mu \mathrm{L}), 2.5 \mathrm{mM}$ dNTPs $(2 \mu \mathrm{L})$, each of $5 \mu \mathrm{M}$ primer $(0.8 \mu \mathrm{L})$, FastPfu
Polymerase $(0.4 \mu \mathrm{L})$ and $10 \mathrm{ng}$ of template DNA. The PCR amplicons were then purified by the AxyPrep DNA Gel Extraction Kit (Axygen Biosciences, USA) and quantified by the QuantiFluor ${ }^{\mathrm{Tm}}-\mathrm{ST}$ fluorometer (Promega Corporation, USA). The final equimolar pool was sequenced (paired end, $2 \times 300-\mathrm{bp}$ ) using Illumina Miseq sequencing technology (Illumina, San Diego, USA).

\section{Bioinformatics processing and analysis of 16S rRNA gene sequence}

Raw FastQ files were demultiplexed, quality-filtered by Trimmomatic, and merged using FLASH. The following standards were applied in data processing: 1) Reads were trimmed with an quality score lower than 20 over a 50bp sliding window; 2) The primers were completely matched, but 2 mismatched nucleotides were allowed [70], and sequence reads with ambiguous bases were excluded from downstream analysis; 3) Sequences containing overlaps of $>10 \mathrm{bp}$ were merged in accordance with their overlaps. Finally, remaining sequences were clustered into operational taxonomic units (OTUs) at $97 \%$ similarity level using UPARSE (version 7.1, http://drive5. com/uparse/). The Taxonomies of each $16 \mathrm{~S}$ rRNA gene sequence was analyzed using the RDP Classifier Algorithm (http://rdp.cme.msu.edu/) at a confidence threshold of 70\% [71] against the Silva (SSU132) 16S rRNA database (Release132 http://www.arb-silva.de).

OTUs counts were normalized before testing groupsignificant taxa and rarified to an equal number of 28,277 sequencing reads per each sample before calculating diversity indices. Sequence data were analyzed using the Quantitative Insights Into Microbial Ecology platform (QIIME; V1.9.1) [72] and R packages (v3.2.0), and the free online Majorbio I-Sanger Cloud Platform (www.i-sanger.com). $\alpha$-diversity were measured using the Ace and Chao indices for richness and using the Shannon and Invsimpson for diversity by Wilcoxon test on the rarefied OTU data. $\beta$ diversity (between-habitat diversity) shows the shared diversity between bacterial population in terms of ecological distance. $\beta$-diversity were calculated by Bray-Curtis dissimilarity algorithms on the rarefied OTU data, and reported according to PCoA with 95\% confidence interval (CI) ellipses. Statistical differences in $\beta$-diversity metrics between $\mathrm{AD}$ group and $\mathrm{NC}$ group were detected using permutational multivariate analysis of variance (PERMANOVA) [73] by the $R$ "vegan" package. Statistically significant differences in specific bacterial taxa between $\mathrm{AD}$ group and $\mathrm{NC}$ group were analyzed using linear discriminant analysis (LDA) effect size (LEfSe) [74]. LDA scores were calculated by LDA effect size. Difference with LDA values $>2.5$ at $P$ value $<0.05$ were considered significant. 


\section{Metabolomics profiling based on UPLC-MS}

Fecal samples $(50 \mathrm{mg})$ were added with $400 \mu \mathrm{L}$ methanol-water $(4: 1, \mathrm{v} / \mathrm{v})$ and the supernatant was obtained according to the following procedure: $6 \mathrm{~min}$ for homogenization, $30 \mathrm{~min}$ for ultrasonically extraction on ice, $30 \mathrm{~min}$ for keeping at $-20^{\circ} \mathrm{C}$ and $15 \mathrm{~min}$ for centrifugation at $13000 \mathrm{rpm}$ at $4{ }^{\circ} \mathrm{C}$. Next, $200 \mu \mathrm{L}$ supernatant was used for UPLC-MS analysis and a mixture of all extraction aliquots was used as a quality control (QC) sample. LC-MS-based fecal metabolic profiling were performed on an Ethylene Bridged Hybrid C18 column $(100 \mathrm{~mm} \times 2.1 \mathrm{~mm}$.id., $1.7 \mu \mathrm{m}$ internal diameter, Waters Corp., Milford, USA), coupled to a Triple TOF $^{\mathrm{ma}} 5600$ mass spectrometer system (AB SICEX, USA). The column temperature was $40{ }^{\circ} \mathrm{C}$. Injection volume of the prepared sample was $20 \mu \mathrm{L}$. The mobile phase included solvent A (water containing $0.1 \%(\mathrm{v} / \mathrm{v})$ formic acid) and solvent B (acetonitrile/isopropanol (1:1) containing 0.1\% (v/v) formic acid) and the flow rate was $0.40 \mathrm{~mL} / \mathrm{min}$. The elution gradient was $5 \% \mathrm{~B}-20 \% \mathrm{~B}$ from 0 to $3 \mathrm{~min}$, $20 \%$ B-95\% B from 3 to $9 \mathrm{~min}$, holding at $95 \%$ B till $13.0 \mathrm{~min}$, then decreased to $5 \% \mathrm{~B}$ in $0.1 \mathrm{~min}$, and holding at $5 \% \mathrm{~B}$ over13.1-16 min. Both positive and negative ion scanning modes were implemented for MS signal acquisition. To obtain information regarding system repeatability, QC samples were injected at every 8 analytical samples throughout the analytical run.

\section{Metabolomics data analysis and metabolite identification}

The raw MS data were processed by Progenesis QI data analysis software (Waters Corp, Milford, MA, USA). Data matrix including peak intensity, retention time of compounds and mass-to-charge ratio were obtained. The detail of data matrix processing procedure was described in our previous published paper [75]. The Data matrix were $\log 10$ transformed for subsequent analysis. Data analysis were performed at Majorbio I-Sanger Cloud platform (www.i-sanger.com). The orthogonal partial least-squares discriminant analysis (OPLS-DA) was applied to detect variation between $\mathrm{AD}$ group and NC group. Although the significant FDR-adjusted $P$ value for metabolites between two groups were not observed (data not shown), we combined univariate methods that analyze each metabolite separately and multivariate OPLS-DA methods to reduce false positive results and improve the reliability of the results [76]. Based on the OPLS-DA analysis, metabolites with variable importance in the projection (VIP) $>1.0, P$ values of $<0.05$ (Student's t-test) referring to published paper [77] were considered to be significantly differed between the $\mathrm{AD}$ and $\mathrm{NC}$ groups. Fold-change (FC) of metabolite in $\mathrm{AD}$ group compared with $\mathrm{NC}$ group were analyzed using the data before $\log 10$ transformation. Metabolites were identified using the METLIN online database (https:// metlin.scripps.edu/) and HDBM database. The molecular pathways and biological functions of differential metabolites were predicted by KEGG pathway database and HDBM database.

\section{Cytokine assay}

IL-6, IL-8, IL-10, and G-CSF were detected using the CBA Human Cytokine Kit (BD Biosciences, San Jose, CA) and IL-7, IL-8, IL- 12p70, IFN- $\gamma$, MCP-1, MIP-1 $\beta$, TNF- $\alpha$ were detected using Bio-Plex Pro Human Cytokine kit (Bio-Rad, Hercules, California). CBA kit assay was performed on BD Accuri C6 flow cytometer (BD Biosciences) according to the manufacturer's instructions and the data analysis was done by using BD CBA Software to generate graphical and tabular formats. The set of calibrators was applied to create the standard curves, and the results were obtained from test samples. For Bio-Plex kit assay, serum samples were diluted 1:1 for measurement, and $50 \mu \mathrm{L}$ of the diluted samples was added to each well. Data were obtained by a Bio-Plex 200 system (Bio-Rad) and analyzed with the Bio-Plex Manager Software Version 5.0.

\section{Statistical analysis}

For comparisons of demographic and clinical data between groups, continuous variables are described with median or interquartile range (IQR), and compared between groups using Mann-Whitney U test. Categorical variables were analyzed by Chi-square test. Serum concentrations of cytokines were compared between $\mathrm{AD}$ group and NC group, using the Mann-Whitney U test. All these statistical analyses were performed using SPSS version 21.0 (SPSS, Chicago, IL, US). A two-sided Pvalue of $<0.05$ was considered to be statistically significant. For correlation analysis, Pearson correlation test using the $\mathrm{R}$ package was performed to calculate the exact correlation coefficient and the corresponding $P$ value (set to 0.05 ) and the Heatmap plots were generated using the $\mathrm{R}$ packages. A binary logistic regression with backward selection was performed to determine which combination of fecal markers were best relevant to predict AD. Receiver operating characteristic (ROC) analyses were performed using STATA version 15.0. The sensitivity, specificity and the area under the ROC curve (AUC) were calculated to evaluate the usefulness of the model for identification of the presence of AD.

\section{Abbreviations}

AD: Alzheimer's disease; NC: Cognitively normal control; $A \beta$ : Amyloid- $\beta$; GMB: Gut microbiota; MCl: Mild cognitive impairment; rRNA: Ribosomal RNA; BBB: Blood-brain barrier; PCR: Polymerase Chain Reaction; SRA: Sequence

Read Archive; OTUs: Operational taxonomic units; PCoA: Principal COordinates analysis; PERMANOVA: Permutational multivariate analysis of variance; LEfSe: Linear discriminant analysis effect size; QC: Quality control; OPLS-DA: Orthogonal partial least-squares discriminant analysis; SD: Standard deviation; ROC: Receiver operating characteristic; AUC: Area under the 
receiver operating characteristic curve; PUFAs: Protecting polyunsaturated fatty acids; APP: Amyloid precursor protein

\section{Supplementary Information}

The online version contains supplementary material available at https://doi. org/10.1186/s12866-021-02286-z.

Additional file 1: Fig. S1. The OUTS distribution and the control analyses of global microbial phenotypes.

Additional file 2: Table S1. a-phylogenetic diversity analysis.

Additional file 3: Table S2. Linear discriminant analysis effect size (LEfSe) analysis for differential bacteria between AD group and NC group.

Additional file 4: Table S3. The coefficient of correlation between microbial genus and fecal metabolites, clinical parameters and inflammatory cytokines.

Additional file 5: Table S4. The $P$ valued of correlation between microbial genus and fecal metabolites, clinical parameters and inflammatory cytokines.

Additional file 6: Table S5. The coefficient of correlation between fecal metabolites and clinical parameters and inflammatory cytokines.

Additional file 7: Table S6. The $P$ valued of correlation between fecal metabolites, clinical parameters and inflammatory cytokines.

\section{Acknowledgements}

The authors thank all the study coordinators, research nurses, psychometrists, and lab technicians for their efforts to this study, and all the participants for their cooperation.

\section{Authors' contributions}

QX and DD made study conception and design. JX, HZ, RW, WW and ZX conducted the acquisition of data and fecal and blood samples. QZ and ZH carried out the clinical diagnosis and interpretation of the data. JX, QX, FS and $\mathrm{XL}$ performed the bioinformatics, statistical analysis, and interpreted data. QX wrote the manuscript. HF and DD revised the manuscript. All authors read and approved the final manuscript.

\section{Funding}

This work was supported by grants from Shanghai Municipal Committee for Health and Family Planning (Award number: GWV-10.1-XK14) and Scientific Research Starting Foundation from Fudan university (to QX), National Key Research and Development Program of China (Grant No. 2018YFC2002000\&2018YFC2002001), National Chronic Disease Project (2016YFC1306402), Scientific Research Plan Project of Shanghai Science and Technology Committee [17411950701, 17411950106], and Shanghai Municipal Science and Technology Major Project [2018SHZDZX01].

\section{Availability of data and materials}

The raw data of $16 \mathrm{~S}$ rRNA gene sequence are available from the Sequence Read Archive (SRA) database of National Center for Biotechnology Information (NCBI) official website (accession number: SRP252374).

\section{Declarations}

Ethics approval and consent to participate

The study protocol was approved by the Medical Ethics Committee of Huashan Hospital at Fudan University (IRB\#2009-195) and the Ethics Committee of the Department of Public Health at Fudan University (IRB\#2019-04-0739), Shanghai, China. All participants, or their legally acceptable representative, provided written informed consent to participate in this study.

\section{Consent for publication}

Not applicable.

\section{Competing interests}

The authors declare no conflict of interest.

\section{Author details}

${ }^{1}$ Department of Preventive Medicine and Health Education, School of Public Health, Fudan University, Shanghai 200032, China. ${ }^{2}$ Institute of Neurology, Huashan Hospital, Fudan University, Shanghai 200040, China. ${ }^{3}$ National Clinical Research Center for Aging Diseases, Shanghai 200040, China.

Received: 20 August 2020 Accepted: 26 July 2021

Published online: 12 August 2021

\section{References}

1. Querfurth HW, LaFerla FM. Alzheimer's disease. N Engl J Med. 2010;362(4): 329-44.

2. Benakis C, Brea D, Caballero S, Faraco G, Moore J, Murphy M, et al. Commensal microbiota affects ischemic stroke outcome by regulating intestinal gammadelta T cells. Nat Med. 2016;22(5):516-23.

3. Dinan TG, Cryan JF. Gut instincts: microbiota as a key regulator of brain development, ageing and neurodegeneration. J Physiol. 2017;595(2):489503.

4. Sampson TR, Debelius JW, Thron T, Janssen S, Shastri GG, Ilhan ZE, et al. Gut microbiota regulate motor deficits and Neuroinflammation in a model of Parkinson's disease. Cell. 2016;167(6):1469-80.

5. Sharon G, Sampson TR, Geschwind DH, Mazmanian SK. The central nervous system and the gut microbiome. Cell. 2016;167(4):915-32.

6. Wang $Y$, Kasper $\mathrm{LH}$. The role of microbiome in central nervous system disorders. Brain Behav Immun. 2014;38:1-12.

7. Zheng P, Zeng B, Liu M, Chen J, Pan J, Han Y, et al. The gut microbiome from patients with schizophrenia modulates the glutamate-glutamine-GABA cycle and schizophrenia-relevant behaviors in mice. Sci Adv. 2019;5: eaau83172.

8. Vogt NM, Kerby RL, Dill-McFarland KA, Harding SJ, Merluzzi AP, Johnson SC, et al. Gut microbiome alterations in Alzheimer's disease. Sci Rep. 2017;7(1): 13537.

9. Park JY, Choi J, Lee Y, Lee JE, Lee EH, Kwon HJ, et al. Metagenome analysis of bodily microbiota in a mouse model of Alzheimer disease using Bacteriaderived membrane vesicles in blood. Exp Neurobiol. 2017;26(6):369-79.

10. Xin Y, Diling C, Jian Y, Ting L, Guoyan H, Hualun $L$, et al. Effects of oligosaccharides from Morinda officinalis on gut microbiota and Metabolome of APP/PS1 transgenic mice. Front Neurol. 2018;9:412.

11. Saji N, Niida S, Murotani K, Hisada T, Tsuduki T, Sugimoto T, et al. Analysis of the relationship between the gut microbiome and dementia: a crosssectional study conducted in Japan. Sci. Rep. 2019;9(1):1-9.

12. Li B, He Y, Ma J, Huang P, Du J, Cao L, et al. Mild cognitive impairment has similar alterations as Alzheimer's disease in gut microbiota. Alzheimers Dement. 2019;15:1357-66.

13. Harach T, Marungruang N, Duthilleul N, Cheatham V, Mc CK, Frisoni G, et al. Reduction of Abeta amyloid pathology in APPPS1 transgenic mice in the absence of gut microbiota. Sci Rep. 2017;7:41802.

14. Borody TJ, Paramsothy S, Agrawal G. Fecal microbiota transplantation: indications, methods, evidence, and future directions. Curr Gastroenterol Rep. 2013;15(8):337.

15. Huttenhower CG. Structure, function and diversity of the healthy human microbiome. Nature. 2012:486(7402):207-14.

16. Chow J, Lee SM, Shen Y, Khosravi A, Mazmanian SK. Host-bacterial symbiosis in health and disease. Adv Immunol. 2010;107:243-74.

17. Cryan JF, Dinan TG. Mind-altering microorganisms: the impact of the gut microbiota on brain and behaviour. Nat Rev Neurosci. 2012;13(10):701-12.

18. Collins SM, Surette M, Bercik P. The interplay between the intestinal microbiota and the brain. Nat Rev Microbiol. 2012;10(11):735-42.

19. Mayer EA. Gut feelings: the emerging biology of gut-brain communication. Nat Rev Neurosci. 2011;12(8):453-66.

20. Cryan JF, O'Mahony SM. The microbiome-gut-brain axis: from bowel to behavior. Neurogastroenterol Motil. 2011;23(3):187-92.

21. Yarza P, Yilmaz P, Pruesse E, Glockner FO, Ludwig W, Schleifer KH, et al. Uniting the classification of cultured and uncultured bacteria and archaea using 16S rRNA gene sequences. Nat Rev Microbiol. 2014;12(9):635-45.

22. Jain A, Li XH, Chen WN. An untargeted fecal and urine metabolomics analysis of the interplay between the gut microbiome, diet and human metabolism in Indian and Chinese adults. Sci Rep. 2019;9(1):9113-91.

23. Zierer J, Jackson MA, Kastenmuller G, Mangino M, Long T, Telenti A, et al. The fecal metabolome as a functional readout of the gut microbiome. Nat Genet. 2018;50(6):790-5. 
24. Quigley E. Microbiota-brain-gut Axis and neurodegenerative diseases. Curr Neurol Neurosci Rep. 2017;17(12):94.

25. Pistollato F, Sumalla CS, Elio I, Masias VM, Giampieri F, Battino M. Role of gut microbiota and nutrients in amyloid formation and pathogenesis of Alzheimer disease. Nutr Rev. 2016;74(10):624-34.

26. Wang $X$, Sun G, Feng T, Zhang J, Huang $X$, Wang T, et al. Sodium oligomannate therapeutically remodels gut microbiota and suppresses gut bacterial amino acids-shaped neuroinflammation to inhibit Alzheimer's disease progression. Cell Res. 2019;29(10):787-803.

27. Stewart CJ, Mansbach JM, Wong MC, Ajami NJ, Petrosino JF, Camargo CJ, et al. Associations of nasopharyngeal Metabolome and microbiome with severity among infants with bronchiolitis. A Multiomic analysis. Am J Respir Crit Care Med. 2017;196(7):882-91.

28. Zheng $P$, Li Y, Wu J, Zhang H, Huang $Y$, Tan $X$, et al. Perturbed microbial ecology in myasthenia gravis: evidence from the gut microbiome and fecal Metabolome. Adv Sci. 2019;6(18):1901441.

29. Akoglu H. User's guide to correlation coefficients. Turk J Emerg Med. 2018; 18(3):91-3.

30. Chi L, Mahbub R, Gao B, Bian X, Tu P, Ru H, et al. Nicotine alters the gut microbiome and metabolites of gut-brain interactions in a sex-specific manner. Chem Res Toxicol. 2017;30(12):2110-9.

31. Qin J, Li Y, Cai Z, Li S, Zhu J, Zhang F, et al. A metagenome-wide association study of gut microbiota in type 2 diabetes. Nature. 2012; 490(7418):55-60

32. Rawlings AM, Sharrett AR, Schneider AL, Coresh J, Albert M, Couper D, et al. Diabetes in midlife and cognitive change over 20 years: a cohort study. Ann Intern Med. 2014;161(11):785-93.

33. Ott A, Stolk RP, van Harskamp F, Pols HA, Hofman A, Breteler MM. Diabetes mellitus and the risk of dementia: the Rotterdam study. Neurology. 1999; 53(9):1937-42

34. de la Monte SM, Wands JR. Review of insulin and insulin-like growth factor expression, signaling, and malfunction in the central nervous system: relevance to Alzheimer's disease. J Alzheimers Dis. 2005;7(1):45-61.

35. Yahr TL, Vallis AJ, Hancock MK, Barbieri JT, Frank DW. ExoY, an adenylate cyclase secreted by the Pseudomonas aeruginosa type III system. Proc Natl Acad Sci U S A. 1998;95(23):13899-904.

36. Ochoa CD, Alexeyev M, Pastukh V, Balczon R, Stevens T. Pseudomonas aeruginosa exotoxin $Y$ is a promiscuous cyclase that increases endothelial tau phosphorylation and permeability. J Biol Chem. 2012;287(30):25407-18.

37. Rufián JS, Macho AP, Corry DS, Mansfield JW, Ruiz-Albert J, Arnold DL, et al. Confocal microscopy revealsin planta dynamic interactions between pathogenic, avirulent and non-pathogenicPseudomonas syringae strains. Mol Plant Pathol. 2018;19(3):537-51.

38. Sokol H, Pigneur B, Watterlot L, Lakhdari O, Bermudez-Humaran LG, Gratadoux JJ, et al. Faecalibacterium prausnitzii is an anti-inflammatory commensal bacterium identified by gut microbiota analysis of Crohn disease patients. Proc Natl Acad Sci U S A. 2008;105(43):16731-6.

39. Louis P, Flint HJ. Diversity, metabolism and microbial ecology of butyrateproducing bacteria from the human large intestine. FEMS Microbiol Lett. 2009;294(1):1-8.

40. Machiels K, Joossens M, Sabino J, De Preter V, Arijs I, Eeckhaut V, et al. A decrease of the butyrate-producing species Roseburia hominis and Faecalibacterium prausnitzii defines dysbiosis in patients with ulcerative colitis. Gut. 2014;63(8):1275-83.

41. Prosberg $M$, Bendtsen F, Vind I, Petersen AM, Gluud LL. The association between the gut microbiota and the inflammatory bowel disease activity: a systematic review and meta-analysis. Scand J Gastroenterol. 2016;51(12): 1407-15.

42. Karlsson FH, Tremaroli V, Nookaew I, Bergstrom G, Behre CJ, Fagerberg B, et al. Gut metagenome in European women with normal, impaired and diabetic glucose control. Nature. 2013;498(7452):99-103.

43. Balamurugan R, Rajendiran E, George S, Samuel GV, Ramakrishna BS. Realtime polymerase chain reaction quantification of specific butyrateproducing bacteria, Desulfovibrio and enterococcus faecalis in the feces of patients with colorectal cancer. J Gastroenterol Hepatol. 2008;23(8 Pt 1): 1298-303.

44. Assa A, Butcher J, Li J, Elkadri A, Sherman PM, Muise AM, et al. Mucosaassociated lleal microbiota in new-onset pediatric Crohn's disease. Inflamm Bowel Dis. 2016;22(7):1533-9.

45. Hansen R, Russell RK, Reiff C, Louis P, Mclntosh F, Berry SH, et al. Microbiota of de-novo pediatric IBD: increased Faecalibacterium prausnitzii and reduced bacterial diversity in Crohn's but not in ulcerative colitis. Am J Gastroenterol. 2012;107(12):1913-22.

46. Lopez-Siles M, Martinez-Medina M, Abella C, Busquets D, Sabat-Mir M, Duncan $\mathrm{SH}$, et al. Mucosa-associated Faecalibacterium prausnitzii phylotype richness is reduced in patients with inflammatory bowel disease. Appl Environ Microbiol. 2015;81(21):7582-92.

47. Chakrabarty P, Ceballos-Diaz C, Beccard A, Janus C, Dickson D, Golde TE, et al. IFN-gamma promotes complement expression and attenuates amyloid plaque deposition in amyloid beta precursor protein transgenic mice. J Immunol. 2010;184(9):5333-43.

48. Birge SJ. The role of estrogen in the treatment of Alzheimer's disease. Neurology. 1997;48(5 Suppl 7):S36-41.

49. Baldereschi M, Di Carlo A, Lepore V, Bracco L, Maggi S, Grigoletto F, et al. Estrogen-replacement therapy and Alzheimer's disease in the Italian longitudinal study on aging. Neurology. 1998;50(4):996-1002.

50. Garcia-Blanco A, Pena-Bautista C, Oger C, Vigor C, Galano JM, Durand T, et al. Reliable determination of new lipid peroxidation compounds as potential early Alzheimer disease biomarkers. Talanta. 2018;184:193-201.

51. Bradley-Whitman MA, Lovell MA. Biomarkers of lipid peroxidation in Alzheimer disease (AD): an update. Arch Toxicol. 2015;89(7):1035-44.

52. Teixeira A, Cox RC, Egmond MR. Furan fatty acids efficiently rescue brain cells from cell death induced by oxidative stress. Food Funct. 2013;4(8): 1209-15.

53. Hanus L, Shohami E, Bab I, Mechoulam R. N-acyl amino acids and their impact on biological processes. Biofactors. 2014;40(4):381-8.

54. Han B, Wright R, Kirchhoff AM, Chester JA, Cooper BR, Davisson VJ, et al. Quantitative LC-MS/MS analysis of arachidonoyl amino acids in mouse brain with treatment of FAAH inhibitor. Anal Biochem. 2013:432(2):74-81.

55. Laske C, Stellos K, Stransky E, Leyhe T, Gawaz M. Decreased plasma levels of granulocyte-colony stimulating factor (G-CSF) in patients with early Alzheimer's disease. J Alzheimers Dis. 2009;17(1):115-23.

56. Tsai KJ, Tsai YC, Shen CK. G-CSF rescues the memory impairment of animal models of Alzheimer's disease. J Exp Med. 2007;204(6):1273-80.

57. Kawada H, Takizawa S, Takanashi T, Morita Y, Fujita J, Fukuda K, et al. Administration of hematopoietic cytokines in the subacute phase after cerebral infarction is effective for functional recovery facilitating proliferation of intrinsic neural stem/progenitor cells and transition of bone marrowderived neuronal cells. Circulation. 2006;113(5):701-10.

58. Shyu WC, Lin SZ, Yang HI, Tzeng YS, Pang CY, Yen PS, et al. Functional recovery of stroke rats induced by granulocyte colony-stimulating factorstimulated stem cells. Circulation. 2004;110(13):1847-54.

59. Schneider A, Kruger C, Steigleder T, Weber D, Pitzer C, Laage R, et al. The hematopoietic factor G-CSF is a neuronal ligand that counteracts programmed cell death and drives neurogenesis. J Clin Invest. 2005;115(8):2083-98.

60. Baruch K, Rosenzweig N, Kertser A, Deczkowska A, Sharif AM, Spinrad A, et al. Breaking immune tolerance by targeting Foxp3(+) regulatory T cells mitigates Alzheimer's disease pathology. Nat Commun. 2015;6:7967.

61. Ishikawa R, Bigley NJ. Sex hormone modulation of interferon (IFN) alpha/ beta and gamma production by mouse spleen cell subsets following picornavirus infection. Viral Immunol. 1990;3(3):225-36.

62. Correale J, Arias M, Gilmore W. Steroid hormone regulation of cytokine secretion by proteolipid protein-specific CD4+ T cell clones isolated from multiple sclerosis patients and normal control subjects. J Immunol. 1998;161(7):3365-74.

63. Qi X, Yun C, Sun L, Xia J, Wu Q, Wang Y, et al. Gut microbiota-bile acidinterleukin-22 axis orchestrates polycystic ovary syndrome. Nat Med. 2019; 25(8):1225-33.

64. Jasarevic E, Howard CD, Misic AM, Beiting DP, Bale TL. Stress during pregnancy alters temporal and spatial dynamics of the maternal and offspring microbiome in a sex-specific manner. Sci Rep. 2017;7:44182.

65. Ding D, Zhao Q, Guo Q, Meng H, Wang B, Yu P, et al. The Shanghai aging study: study design, baseline characteristics, and prevalence of dementia. Neuroepidemiology. 2014;43(2):114-22.

66. American Psychiatric Association. Diagnostic and Statistical Manual of Mental Disorders. 4th ed; 1994. p. 143-7.

67. McKhann G, Drachman D, Folstein M, Katzman R, Price D, Stadlan EM. Clinical diagnosis of Alzheimer's disease: report of the NINCDS-ADRDA work group under the auspices of Department of Health and Human Services Task Force on Alzheimer's disease. Neurology. 1984;34(7):939-44.

68. Ding D, Zhao Q, Guo Q, Meng H, Wang B, Luo J, et al. Prevalence of mild cognitive impairment in an urban community in China: a cross-sectional analysis of the Shanghai aging study. Alzheimers Dement. 2015;11(3):300-9. 
69. Smirnov DA, Morley M, Shin E, Spielman RS, Cheung VG. Genetic analysis of radiation-induced changes in human gene expression. Nature. 2009; 459(7246):587-91.

70. Sipos R, Szekely AJ, Palatinszky M, Revesz S, Marialigeti K, Nikolausz M. Effect of primer mismatch, annealing temperature and PCR cycle number on 165 rRNA gene-targetting bacterial community analysis. FEMS Microbiol Ecol. 2007;60(2):341-50

71. Cole JR, Wang Q, Cardenas E, Fish J, Chai B, Farris RJ, et al. The ribosomal database project: improved alignments and new tools for rRNA analysis. Nucleic Acids Res. 2009;37(Database issue):D141-5.

72. Caporaso JG, Kuczynski J, Stombaugh J, Bittinger K, Bushman FD, Costello EK, et al. QIIME allows analysis of high-throughput community sequencing data. Nat Methods. 2010;7(5):335-6.

73. McArdle B, Anderson M. Fitting multivariate models to community data: a comment on distance-based redundancy analysis. Ecology. 2001:82:290-7.

74. Segata N, Izard J, Waldron L, Gevers D, Miropolsky L, Garrett WS, et al. Metagenomic biomarker discovery and explanation. Genome Biol. 2011; 12(6): $: 60$.

75. Zhu H, Abdullah AS, He J, Xi J, Mao Y, Feng Y, et al. Untargeted urinary metabolomics and Children's exposure to secondhand smoke: the influence of individual differences. Int J Environ Res Public Health. 2021;18(2):710.

76. Anderson JR, Phelan MM, Clegg PD, Peffers MJ, Rubio-Martinez LM. Synovial fluid metabolites differentiate between septic and nonseptic joint pathologies. J Proteome Res. 2018;17(8):2735-43.

77. Li J, Zhao F, Wang Y, Chen J, Tao J, Tian G, et al. Gut microbiota dysbiosis contributes to the development of hypertension. Microbiome. 2017;5(1):1-9.

\section{Publisher's Note}

Springer Nature remains neutral with regard to jurisdictional claims in published maps and institutional affiliations.

Ready to submit your research? Choose BMC and benefit from:

- fast, convenient online submission

- thorough peer review by experienced researchers in your field

- rapid publication on acceptance

- support for research data, including large and complex data types

- gold Open Access which fosters wider collaboration and increased citations

- maximum visibility for your research: over $100 \mathrm{M}$ website views per year

At $\mathrm{BMC}$, research is always in progress.

Learn more biomedcentral.com/submissions 